\title{
The retention of responses to individual stimuli and stimulus classes'
}

\author{
WILLIAM E. MONTAGUE AND ALEXANDER J. WEARING \\ UNIVERSITY OF ILLINOIS
}

Items in a paired-associate task that may be encoded into concept classes are better remembered than items which must be coded individually. Three groups, one with 8 words and 8 responses, a second with 32 words and 8 responses, and a third with 32 words and 16 responses learned to a criterion of three correct trials, and after a seven day retention interval, were tested for recall. Although there were no differences in the error scores, the second group (32 words and 8 responses) showed shorter latencies during acquisition, and less increase of latencies over the retention interval. These differences were attributed to the greater number of effective repetitions per response, which was mediated by conceptual encoding of the stimulus words. It was suggested that a minimum of three instances is necessary to mediate conceptual encoding in tasks of this nature.

Items in a paired associate (PA) task that may be encoded into concept classes are better remembered than items which must be coded individually (Hunt, 1962). Thus, the set of words chair, table, desk, when each is paired with the same response, will be learned more readily than apple, iron, desk. The usual explanation for this phenomenon is that in the first case, the association that is learned is between the class name, "furniture," and the response, rather than between the response and the individual items.

From this point of view, each instance of the concept class is equivalent to a repetition of the $S-R$ pair, and so a list of 30 items, divisible into 10 concepts, should be retained as well, or better, than 10 unrelated S-R pairs. Lloyd (1960) tested the hypothesis that there would be no difference in retention between $\mathrm{Ss}$ who learned responses to specific stimull and $\mathrm{Ss}$ who learned responses to classes of stimuli if the number of response units was the same in both situations. He found that 8 words associated with 8 numbers, and 24 words which could be organized into 8 concept classes were both equally well retained.

To control for number of repetitions the 8-pair group was given three exposures of each pair on a trial while Ss learning the 24-word list saw each pair once. Since each concept class occurred three times in the latter list, the number of repetitions for each concept class was the same as that for individual pairs in the 8-word list.

The first aim of the present experiment was to partially replicate Lloyd's study; only item (individual S-R pairs) rather than concept exposure was kept constant across groups. There were two groups of Ss, one with 8 words and 8 responses (the 8-8 group) and another with 32 words and 8 responses (the 32-8 group). The effect of this change was to give the mediators in the 32-8 group four times as many repetitions as in the 8-8 group. Consequently, it was predicted that the retention performance of the 32-8 group would be superior to that of the 8-8 group.

In addition, to ascertain the effect of number of responses on retention, a group of Ss had to learn 32 words paired with 16 responses (the 32-16 group). Since fewer instances of each concept class were given and since retention load is greater, it was predicted that their recall should be inferior to that of the 32-8 group, even though the list length of both groups was the same.

Method

Three groups (total $N=60$ ) of undergraduate Ss learned word (noun)-letter pairs. Two groups were similar to those used by Lloyd (1960). One group (8-8) learned 8 stimuli and 8 responses while another (32-8) learned 32 stimuli which could be grouped into 8 classes, each with 4 words per response. A third (32-16) group learned 32 stimuli which consisted of 16 classes, each with two words per response. The list for the first group was 8 pairs long and for the second and third groups the lists were 32 pairs long.

Items were presented automatically on a CRT display in front of $S$ for 5 sec. A modified recall procedure developed by Battig (1965) was used. All the pairs were presented singly and then each stimulus was presented in a different order to test recall. Items not correctly recalled were presented and tested again. This procedure was repeated until all items were correctly recalled, ending a trial. Then the entire list was presented again and tested to start another trial. Three such trials were given so that all pairs had been given correctly three times prior to the retention interval. Ss typed their responses on a keyboard directly linked to a computer. All responses and response times were automatically recorded.

Immediately after attaining criterion, Ss were asked to respond, in writing, to a questionnaire. Only stimuli were shown on the display, singly, and, on a data sheet, $S$ wrote both the response (if he recalled it) and any associative device he used in learning the pair. After the questionnaire, another test trial was given to the $\mathrm{Ss}$, in which all stimuli were presented one at a time to test final performance level. One week later the Ss were tested for recall.

Results and Diseussion

The proportion of items correct on each test trial is shown in Fig. 1. The only significant difference be- 
tween groups occurs on the first test trial. This proportion represents the number correct on the initial test trial within a trial immediately after the entire list has been presented for the first time. All groups performed nearly perfectly. The error scores suggest virtually all-or-none learning. Once each item was responded to correctly once, performance was almost perfect. Trials 2 and 3 were, in effect, overlearning trials, and although the levels of recall a week later are in the expected order, the differences are not significant.

The latency results shown in Fig. 2 modify the allor-none interpretation, however. Although the proportion correct on the last three acquisition tests is nearly perfect, latencies continue to decrease. Moreover, the 32-8 group had significantly shorter latencies during acquisition $(p<.05)$, and the increase in latencies over the retention period was significant $(p<.01)$. In addition, all comparisons between these increases for the groups were significant $(p<.05)$ with the 32-16 group showing the longest latencies. Clearly, the 32-8 group is superior at all points.

The faster latencies at retention of the 32-8 group may be attributed to the greater number of effective repetitions per response. Four items were paired with each response term for that group, while only two were paired for the 32-16 group, and only one per response term for the 8-8 group. The superiority of the 32-8 group may be attributed to the conceptual encoding.

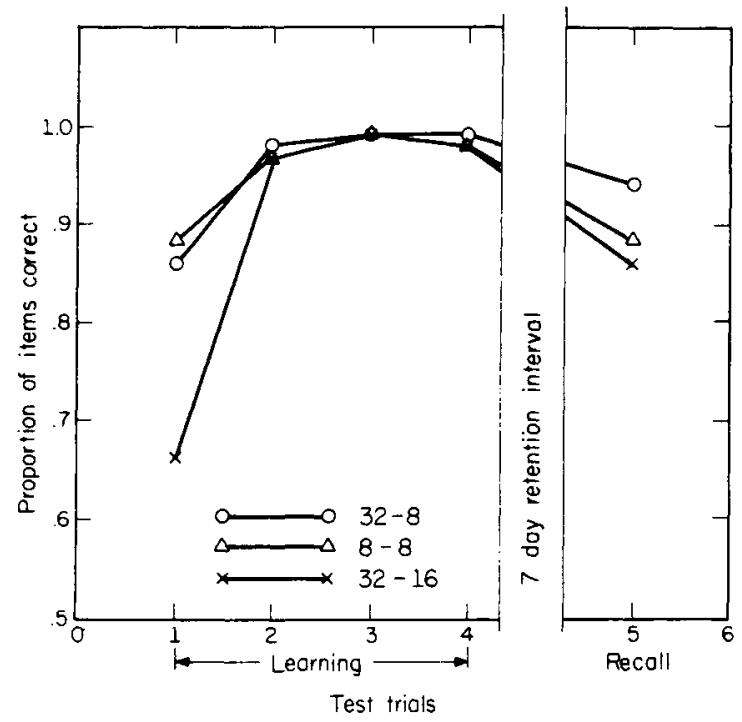

Fig. 1. Proportion of items conrect on test trials during learning and recall.

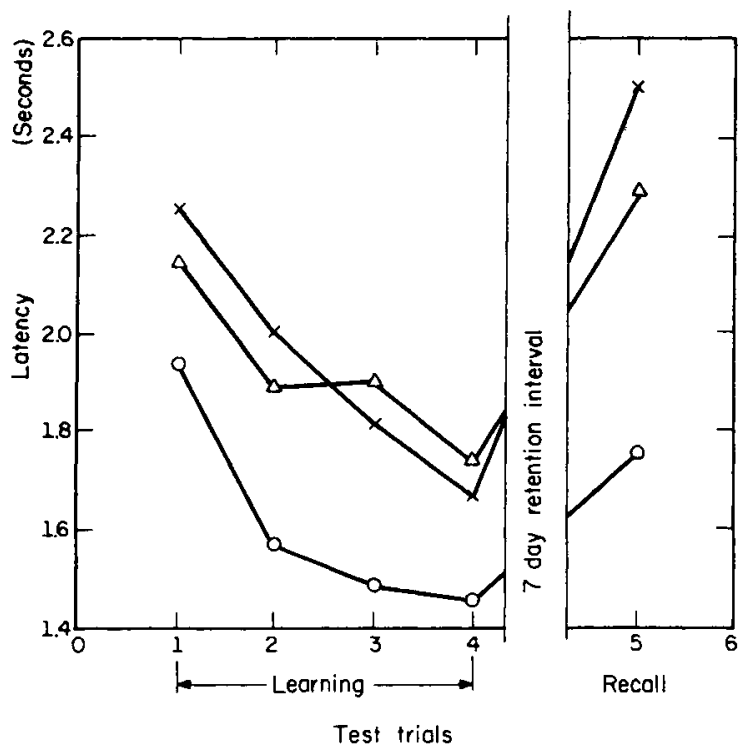

Fig. 2. Latencies for correct responses on test trials during learning and recall.

Confirmation for this view comes from a comparison of the 32-8 and the 32-16 groups. Both had the same number of items in their lists, but the 32-16 group had twice as many concepts, and was inferior, having both more errors and longer latencies.

The faster latencies of the 32-8 group, even at the beginning of acquisition, suggests that the third and fourth instances of each concept may have been equiralent to extra repetitions. That is, once the concept is identified or learned, any further instances only contribute to its overlearning. The differences in performance between the 32-8 and the 32-16 groups suggest that the critical number of instances may be about three. Two Instances were not enough to allow all the concepts in the 32-16 group to be learned during the first trial, while four instances (in the 32-8 group) seemed to be more than enough.

\section{References}

BATTIG, W. F. Procedural problems in paired-associate learning research. Psychol Monogr. Suppl., 1965, 1, No. 1.

HUNT, E. B. Concept learning: An information processing problem. New York: Wiley, 1962.

LLOYD, K. E. Retention of responses to stimulus classes and specific stimuli. J. exp. Psychol, 1960, 59, 54-59.

\section{Note}

1. This research was supported in part by the Joint Services Electronics Program (U. S. Army, U. S. Navy and U. S. Air Force) under Contract No. D. A. 28043 AMC 00073 (E). 